Desarrollo sostenible a la tica: Sustainable Development "a la geopolítica y ambiente en la tica": Geopolitics and Administración Figueres Olsen Environment in Figueres Olsen (1994-1998)

RESUMEN Administration (1994-1998)

ABSTRACT

El presente artículo estudia, desde una The following article analyzes from a perspectiva geopolítica decolonial, la va- geopolitical and decolonial approach the riable ambiental en los discursos y accio- environmental variable in the speeches nes de la Administración liberacionista de and actions of the National Liberation José María Figueres Olsen (1994-1998) party government of the former ex-presien Costa Rica, por medio del papel cen- dent José María Figueres Olsen in Costa tral asignado a un «desarrollo sostenible Rica (1994-1998). His government gave a la tica» durante su gobierno, convirtién- a central role to the sustainable developdolo en paradigma en sus discursos y ment, turning it into a unifying force of the acciones tanto internas como externas. discourses and actions concerning both, Se demuestra como su uso se debe ma- his domestic and international public poliyormente al peso geopolítico de las dis- cies. The article demonstrates that this cusiones internacionales sobre la rela- concept is used mostly according to ción sociedad-naturaleza y a una conti- some geopolitical conditions, regarding nuidad del modelo economicista de uso the international discussions about the rede la naturaleza.

Palabras Clave

Desarrollo sostenible; políticas ambientales; racionalidad económica (bio)colonialidad del poder; geopolítica lationship between society and nature within the boundaries of an economic model or theory about nature.

KEY WORDS

Sustainable development; environmental policies; economic rationality; (bio)coloniality of power; geopolitics
Artículo recibido:

1 de mayo del 2014.

Evaluado:

1 de julio del 2014.

Aceptado:

21 de julio del 2014.

Licenciado en Ciencias Políticas de la Universidad de Costa Rica. Asesor técnico regional para el Programa Salud, Trabajo y Ambiente (SALTRA) del Instituto Regional de Estudios en Sustancias Tóxicas (IRET) de la Universidad Nacional de Costa Rica. Miembro del Grupo de trabajo de espacialidad crítica en el pensamiento político-social latinoamericano: nuevas gramáticas de poder, territorialidades en tensión del Consejo Latinoamericano de Ciencias Sociales (CLACSO).

Correo electrónico: cmongehernandez@gmail.com

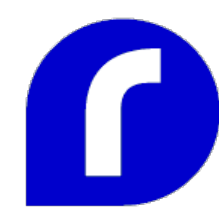

Claudio Monge Hernández 


\section{Desarrollo sostenible a la tica: geopolítica y ambiente en la Administración Figueres Olsen (1994-1998)}

\section{Génesis del «desarrollo sostenible a la tica»}

El desarrollo sostenible se ha posicionado a nivel mundial en menos de 30 años como el principal referente sobre el equilibrio entre las relaciones sociedad-naturaleza, cuya idea se suele ligar a la protección ambiental, constituyéndose en algunos casos casi como el único sinónimo posible para esto. Lo que la convierte en una institución discursiva que monopolizan los principales debates sobre progreso de las sociedades humanas, gracias a que confronta discursivamente al desarrollismo clásico, pero que en la práctica se suma y adorna la estructura del desarrollismo sin modificarlo en su núcleo duro; la economía de crecimiento.

Esta visión de desarrollo, tan ampliamente difundida y asumida por élites y sociedad civil de todo tipo y lugar, se compone de tres elementos, que son centrales en el funcionamiento del llamado sistema internacional moderno, los cuales desde nuestra perspectiva se pueden explicar de esta forma:

- Una racionalidad económica. La cual se traduce en la búsqueda de consolidar modelos de desarrollo basados principalmente en estándares económicos, especialmente el crecimiento económico como sinónimo de progreso global, nacional, local y hasta individual (Castoriadis, 2003; Fotopoulos, 2002; Leff, 1994; Leff, 2004).

- Una lógica que algunos llaman colonial y otros imperialista. No obstante, lo cierto es que ambas van de la mano para promover e impo- 
ner los valores y visión occidental de la historia y el futuro, lo que conlleva una clasificación del mundo según el color de la piel y la función económica en los mundos no occidentales. A esto se le llama más recientemente colonialidad, entendida como historias locales de los centros de poder y élites occidentales que se transforman en diseños globales, pero hay que añadir que es cada unidad de la periférica y semiperférica la que los asume con sus particularidades locales, de ahí su carácter heterárquico y no únicamente jerárquico u horizontal (Quijano, 2000; Mignolo, 2003; Castro-Gomez, 2007).

- Esta colonialidad es imposible si no existen también como (bio)colonialidad. Es decir, como colonización no solo de sociedad humanas por medio de su división racial y económica, sino del complejo y dinámico caleidoscopio de la vida en el planeta por medio de la racionalidad económica mencionada, donde la principal referencia es la visión utilitaria de la naturaleza y su uso es sinónimo de "recurso ambiental" o "capital natural" (Alimonda, 2011; Cajigas-Rotundo, 2011).

En este orden, el nacimiento del desarrollo sostenible globalmente se enmarca en las décadas 1970 y 1980, cuando entra en crisis el llamado diseño socialdemócrata del mundo ${ }^{1}$ por una nueva historia local mundializada por medio del retorno del liberalismo en su forma neoliberal, surgido y promulgado, entre otros/as, por los gobiernos estadounidense de Ronald Reagan (19811989) y británico de Margaret Thatcher (1979-1990), organismos como el Fondo Monetario Internacional (FMI) y Banco Mundial (BM), así como el Foro Económico Mundial (FEM) y plataformas panamericanas del Consenso de Washington y la Comisión Económica para América Latina y el Caribe (CEPAL) con su regionalismo abierto.

No es casualidad que desde estos entes supranacionales, internacionales y transnacionales se dicten las políticas, que bajo excusa de armonía y consenso homogenicen y redefinan constantemente las relaciones internacionales para que estas sean funcionales a la globalización y la internacionalización de la economía de crecimiento por medio del libre comercio. Como consecuencia, el sistema internacional de Estados deja de regirse por el esquema basado de soberanías nacionales de la Paz de Westfalia por uno multinivel, con macro y micro-regiones, así como Estados tradicionales, donde se desnacionaliza/transnacionaliza la producción y comercialización de bienes y la prestación de servicios, lo que conlleva a una reforma de las instituciones globales, regionales, nacionales, y locales (Fotopoulos, 2002; Sassen, 2001).

En lo que respecta a la variable ambiental, es hacia el último cuarto del Siglo $X X$ que se empieza a tomar consciencia del uso, abuso y explotación desmedida de la naturaleza, gracias a aportes como los del Club de Roma con su informe "Los límites del crecimiento" (1972), lo que hace que se posicione el tema dentro del sistema internacional, especialmente en el marco de la ONU,

1. El cual buscaba, entre otras cosas y desde distintas versiones, como el marxismo clásico, el eurocomunismo, la socialdemocracia o el liberalismo keynesiano, un papel protagónico del Estado en el control y regulación de la economía, tanto en su producción como en la prestación de servicios. 
dentro del cual inician discusiones sobre la armonía entre sociedad-naturaleza, pero bajo los conceptos de medio ambiente y desarrollo, primero en la "Conferencia Internacional sobre Medio Ambiente" de Estocolmo (1972) y de manera posterior en la "Conferencia sobre Medio Ambiente y Desarrollo" de Río de Janeiro (1992).

Las expectativas de los países así llamados subdesarrollados sobre los alcances de los compromisos eran optimistas, en especial en lo que respecta al compromiso de las superpotencias para dotar de recursos suficientes a la conservación de la biodiversidad global. No obstante, la resistencia a compromisos concretos fue grande pese al consenso entre los países del sur, por lo que los acuerdos (como todo acuerdo fuera del Consejo de Seguridad de la ONU) son básicamente consultivos (Segura, 1992). Lo que demuestra que cuando lass historias locales de la periferia tratan de influir en los diseños globales de las superpotencias son finalmente estas últimas las que se imponen para no concretar temas que afecten sus historias locales.

Por ejemplo, la Administración Clinton (1993-2001) basó su política exterior en la Doctrina de Ampliación Democrática, la cual tiene como meta consolidar un sistema internacional de democracias de mercado, tanto en América Latina como en las entonces nuevas repúblicas de Europa del Este. Así, se amplió el espectro clásico de la anterior Doctrina de Seguridad Nacional, procurando incluir temáticas como la democracia y el ambiente para favorecer y fortalecer el mercado global (Departamento de Estado, 2000; Hybel y Linch, 1994).

Esta visión, debido al carácter dispar del poder en el sistema internacional, termina por consolidarse a pesar del alcance de las cumbres mencionadas. Como resultado, la racionalidad económica no se cuestiona y lejos de debilitar al diseño neoliberal, los debates se concentran más en la definición semántica que debía tomar el desarrollo entre "sostenido", "sustentable" o "sostenible", que en debatir sobre los contenidos económico-políticos de dichos diseños, como la creciente influencia de los Programas de Ajuste Estructural (PAE) sobre los Estados-nacionales periféricos. Finalmente, se impuso que fuera el adjetivo sostenible el que se usara en la definición de la Conferencia de la ONU en 1989, a saber:

...un nuevo sendero de progreso que permite satisfacer las necesidades y aspiraciones del presente, sin comprometer la capacidad de las futuras generaciones de satisfacer sus propias necesidades (ONU, 1987).

Se trata de una definición abierta y sin compromisos concretos que favorece múltiples interpretaciones para que pueda ser aceptada y asumida por diversos grupos en el mundo, desde empresarios, organizaciones de la sociedad 
civil y gobiernos, tanto de derecha como de izquierda (Estrada y Suárez, 2006; Segura, 1992) que crean sus propias versiones locales. Tal es el caso del «desarrollo sostenible a la tica» de la Administración liberacionista de Figueres Olsen, que se expondrá adelante.

Fue en la "Conferencia de las Naciones Unidas sobre el Medio Ambiente y el Desarrollo" (cc. Conferencia de la Tierra) realizada en Río de Janeiro (1992), donde se lograron los acuerdos y recomendaciones (no obligaciones) más grandes, destacándose el Programa 21 (cc. Agenda 21) por sus pretensiones en diversas áreas, que iban desde las competencias municipales hasta la forma del Derecho como disciplina, intentado ser un motor global para promover el desarrollo sostenible. Pero con el inconveniente clásico del Derecho Internacional, a saber, que su aplicación queda a la voluntad de cada Estado, es decir, de sus élites gobernantes/dominantes.

Veinte años después es posible evaluar la redefinición del desarrollo que se ha institucionalizado en la comprensión de nuestra relación con la biodiversidad. Adriana Gómez Bonilla puntualiza las principales políticas que consolidan el modelo en la era capitalista, divida en dos etapas complementarias en el tiempo (2012: 285-302):

- Capitalismo extractivo (Siglo XIX a la actualidad):

- Explotación forestal.

- Ganadería.

- Minería.

- Capitalismo ecológico (1970 a la actualidad):

- Bioprospección, es decir, el uso comercial de la diversidad biológica.

- Biocombustibles.

- Pago de servicios ambientales.

- Desde el ecoturismo, provisión de alimentos, agua, leña, fibras, recursos genéticos hasta la regulación del clima, el control de plagas, la recarga de mantos acuíferos y el mantenimiento de la fertilidad del suelo, etc.

En esta racionalidad económica sobre la vida que también llamamos (bio)coIonial, se monopolizan las interpretaciones y acciones del desarrollo sostenible que la administración liberacionista de Figueres Olsen va a usar para su «desarrollo sostenible a la tica», paradigma y agente aglutinante del discurso y las prácticas tanto a lo interno como a lo externo de su gobierno. No obstante, se verá que este responde a las dinámicas geopolíticas y geoeconómicas de los años 1970 acá descritas de forma resumida, simultáneamente al 
regreso del liberalismo como diseño global (en su forma de neoliberalismo), lo que definitivamente marcó el contenido de los debates y políticas mencionadas.

\section{Discurso del «desarrollo sostenible a la tica»}

Si bien es durante la Administración de Figueres Olsen (1994-1998) que se consolida la visión dominante del desarrollo sostenible, el «desarrollo sostenible a la tica», fue durante la Administración Arias Sánchez (1986-1990) que se introdujo el concepto por primera vez dentro de la discusión de la Estrategia de Conservación para el Desarrollo Sostenido o Estrategia de Conservación para el Desarrollo Sostenible (ECODES). Hay que destacar que el acrónimo se usó indistintamente sostenido y sostenible (Segura, 1992; Quesada, 1998). Lo anterior debido a que estos debates ocurrieron antes de la Conferencia de la ONU que en 1989 instituyó definitivamente el término "sostenible".

La ECODES buscó, entre otras cosas, introducir la variable ambiental dentro la legislación del país, como el Artículo 50 de la Constitución Política, reformado de la siguiente forma: "Toda persona tiene derecho a un ambiente sano y ecológicamente equilibrado. Por ello, está legitimada para denunciar los actos que infrinjan ese derecho y para reclamar la reparación del daño causado".

No obstante, la operacionalización de la ECODES, por medio de nuevas leyes y códigos, quedó relegada hasta la Administración Figueres Olsen (CueIlo, 2011) cuando introduce su versión propia, el «desarrollo sostenible a la tica», la cual se empieza a delinear desde el mismo traspaso de poderes el 8 de mayo de 1994, con lo que entonces llamó "un nuevo estilo de desarrollo" (Figueres Olsen, 1994a) conformado por:

- La integración de la sociedad, teniendo como ejes centrales al individuo y a la familia.

- El perfeccionamiento de la democracia formal para mejorar la efectividad de la actividad ciudadana.

- Avanzar de una apertura económica a una integración con el mundo.

- Transformación del fondo de las instituciones, especialmente del Estado.

- Abandonar un estilo de desarrollo sin visión a futuro e instituir un desarrollo sostenible. 
El "nuevo estilo de desarrollo" se define como la búsqueda de "un mayor bienestar general en el presente mientras cuidamos los grandes equilibrios que hacen posible nuestro desarrollo en el largo plazo" (MIDEPLAN, 1998, p.299). En esta perspectiva los equilibrios corresponden a: (a) equilibrio político-institucional, que busca la amplitud democrática en la toma de decisiones; (b) equilibrio de la estructura social, que pretende la integración interna; (c) equilibrio económico, para alcanzar largos periodos de progreso material sostenido; (d) equilibrio ambiental, según el cual pretendía una armonía entre la base natural y la vida social (MIDEPLAN, 1998).

Esta definición de desarrollo sostenible, en la cual la extensión en el tiempo es lo principal, ubica al ambiente, semántica y jerárquicamente, de último lugar, dando mayor importancia a los temas económicos y administrativos. Este uso discursivo no es gratuito, ya que la Administración pretendía con este tipo de desarrollo:

Una continuación, porque se trata de profundizar los excepcionales logros alcanzados en la sostenibilidad del sistema político-institucional y del desarrollo social. Una ruptura, porque trata de alejarse de las políticas económicas cortoplacistas que no ayudan a transformar la estructura productiva para crear una base económica sostenible, y se trata también de superar gradualmente las prácticas depredadoras del ambiente (MIDEPLAN, 1998, p.300).

Se observa cómo se hace referencia a una continuidad con respecto a las acciones que ya se vienen aplicando sobre el Estado costarricense, la cual se enmarca dentro del Pacto Figueres-Calderón en 1995, que significa la consolidación del orden bipartidista que converge en el proceso de reforma de Estado y de apertura económica iniciada con los PAE y promovida por organismos internacionales como el FMI y BM (Molina y Palmer, 1997).

Nuevamente se destaca cómo la naturaleza, para esta versión del desarrollismo, siempre queda al final, es decir, queda no solo relegada sino condicionada al cumplimiento previo de los ejes anteriores a este, es decir, a la reforma estatal y a la apertura comercial. En este sentido, cuando se habla de políticas ambientales, Figueres Olsen plantea que dicho modelo no solo busca:

evitar y revertir el deterioro ambiental, sino que también apunta a promover una estructura productiva que desperdicie y dañe menos los recursos y que los valorice más, para construir bases sólidas para integrar la economía costarricense a una economía mundial 
cada vez más abierta y globalizada (MIDEPLAN, 1998, p.303; énfasis propio).

Se puede observar la existencia de un claro dominio de lo económico sobre lo ecológico, ya que pese a la referencia de "evitar y revertir", estas intenciones dependen de la integración de Costa Rica al nuevo esquema económico global posterior a la Guerra Fría, donde tanto Estados Unidos como el neoliberalismo se posicionan uno como potencia y el otro como único diseño global. Para esto, vemos en el mismo discurso que el criterio de conservación de la estructura productiva es que "desperdicie y dañe menos", lo cual no es lo mismo que decir "no desperdicie y no dañe".

Se constituye así el «desarrollo sostenible a la tica», que se puede definir como: una versión del desarrollo sostenible que busca incluir la variable ambiental en las políticas de Estado-nación costarricense, sin interrumpir los procesos de reforma institucional, apertura e integración económica de Costa Rica bajo los estándares del diseño neoliberal como esquema económico-político dominante.

\section{Cuadro 1}

Definiciones de Desarrollo Sostenible

\begin{tabular}{|c|c|c|}
\hline $\begin{array}{l}\text { ONU - Informe } \\
\text { Brundtland }\end{array}$ & $\begin{array}{c}\text { Asamblea Legislativa - } \\
\text { ECODES }\end{array}$ & $\begin{array}{c}\text { Administración Figueres } \\
\text { Olsen }\end{array}$ \\
\hline $\begin{array}{l}\text { Un nuevo sendero de } \\
\text { progreso que permite } \\
\text { satisfacer las necesidades } \\
\text { y aspiraciones del } \\
\text { presente, sin comprometer } \\
\text { la capacidad de las futuras } \\
\text { generaciones de satisfacer } \\
\text { sus propias necesidades. }\end{array}$ & $\begin{array}{l}\text { Un proceso de acción y } \\
\text { manejo de recursos } \\
\text { ambientales, junto a la } \\
\text { ciudadanía y al desarrollo } \\
\text { científico, por medio de } \\
\text { nuevos esquemas legales, } \\
\text { para mejorar las } \\
\text { necesidades básicas sin } \\
\text { perjudicar la calidad de } \\
\text { vida y el desarrollo futuro. }\end{array}$ & $\begin{array}{l}\text { Buscar un mayor bienestar } \\
\text { general en el presente } \\
\text { mientras cuidamos los } \\
\text { grandes equilibrios que } \\
\text { hacen posible nuestro } \\
\text { desarrollo en el largo } \\
\text { plazo. }\end{array}$ \\
\hline
\end{tabular}

Fuente: Elaboración propia

Vemos en el Cuadro 1 que existió una mayor influencia de los conceptos del Informe Brundtland que los de la propia discusión nacional de la ECODES (Stokke y Mármola, 1993), es decir, el «desarrollo sostenible a la tica» no fue tan costarricense como pretendía la Administración liberacionista, al favorecer un concepto que internacionalmente no pudo concretar compromisos más allá de recomendaciones no vinculantes. Situación en la que "es obvio que el verdadero objetivo de este enfoque no propone maneras de lograr el 
desarrollo [sostenible] sino maneras de crear una economía de mercado 'inocua para la ecología', una contradicción de términos" (Fotopoulos, 2002, p.145).

El «desarrollo sostenible a la tica» sirvió, por un lado, de base para su política regional para Centroamérica por medio del proceso de integración del Sistema de Integración Centroamericana (SICA) y, por otro lado, como mediación para las políticas de diplomacia y comercio exterior, una suerte de carta de presentación ante la comunidad internacional. Para lo cual se suscriben acuerdos como la "Declaración de Río sobre el medio ambiente y el desarroIlo", el "Programa 21", la "Convención sobre la diversidad biológica", la "Convención sobre el cambio climático" y la "Declaración sobre principios forestales".

Por tanto, la política exterior costarricense, regional y globalmente, hizo un uso similar del «desarrollo sostenible a la tica» como se hizo al interno, con la naturaleza condicionada a objetivos económico-políticos del diseño neoliberal dominante. Lo anterior para que, entre otras cosas, fuera adoptado por los países centroamericanos por medio del SICA y, al mismo tiempo, sirviera de plataforma para las negociaciones bilaterales y multilaterales del país con otras regiones, organismos internacionales y Estados (Monge, 2013).

Las intenciones para Centroamérica se pueden ejemplificar con la siguiente metáfora utilizada por Figueres Olsen durante la Cumbre Ecológica en Masaya (1994a) cuando afirma que "Aquí si me lo permiten, me quito el sombrero de centroamericano para ponerme mi sombrero de costarricense... [Para luego decir] Me quito mi sombrero de costarricense y sigo con el de centroamericano" (Monge, 1995, p.41).

Los sombreros son una síntesis de cómo la Administración se comporta con la región pese al discurso integracionista, al afirmar tener el "sombrero de centroamericano" hace referencia a las necesidades del SICA y su propuesta del «desarrollo sostenible a la tica», mas dice tener el "sombrero de costarricense" cuando quiere resaltar (discursivamente) lo que él considera resultados positivos de este.

Durante la XV Cumbre de Presidentes Centroamericanos en Guácimo de Limón, el presidente Figueres Olsen afirma que su concepto de desarrollo sostenible es el modelo necesario para avanzar y consolidar la pacificación y democratización del proceso de Esquipulas en un modelo de progreso compatible tanto con las exigencias del orden mundial como con las necesidades regionales, para:

Evitar los estilos de desarrollo nacional que se caracterizan por producir y vivir en el presente, sin preocuparse por el futuro. No podemos contentarnos con mejoras frágiles y superficiales de los proble- 
mas nacionales, ni nos pueden satisfacer los logros aparentes de hoy, que nos heredan grandes problemas para el mañana. Mientras resolvemos los problemas más urgentes, necesitamos también crear bases firmes para que las generaciones futuras puedan vivir mejor que las actuales. Necesitamos avanzar, amigos y amigas, por la vía del Desarrollo Sostenible (Figueres Olsen,1994b, p. 7).

Como se puede ver, un elemento constante de estos discursos es la extensión en el tiempo, pero para entender qué es lo que se quiere transformar y conservar, se debe analizar la retórica usada en la Cumbre Ecológica al enumerar los retos necesarios para Centroamérica (Monge, 1995):

- Ordenamiento territorial en función de parques nacionales, planificación urbana e industrial, agricultura y siembra de árboles para comercio.

- Contribuir con los esfuerzos mundiales sobre el cambio climático, por medio del uso adecuado de los recursos.

- Firma del "Convenio mundial de biodiversidad biológica", principal instrumento de bioprospección.

- Establecimiento de áreas de conservación en función de la protección ambiental con fines turísticos.

- Actualizar los planes de estudios para favorecer a los más pobres y la movilidad social, así como para crear "centroamericanidad" y competir mejor en el mercado global.

- Culturalmente, promover esta visión de conservación ambiental dentro la población.

- Favorecer la producción nacional ante la crisis energética.

- Buscar sistemas de producción para la agricultura que no degraden el ambiente.

- Políticas económicas para usar las señales del mercado y sincronizar el uso adecuado de los recursos naturales.

- Fortalecer el poder de compra del Estado de productos amigables con el ambiental. 
- Tener producción nacional orientada hacia los valores agregados de la región, de forma integrada y eficiente, como el así llamado ecoturismo.

- Participación de la sociedad civil en la toma de decisiones a partir de la aceptación del modelo de desarrollo sostenible.

En conjunto, vemos cómo nuevamente el ambiente depende del cumplimiento previo de temas económicos y administrativos, lo que convierte a la conservación en una forma de lucro más, como dice Figueres, en función de las señales del mercado. Es decir, se plantea conservar el sistema económicopolítico dominante y transformar la estructura productiva para que el primero pueda disponer de la naturaleza siempre en forma de recursos. Asimismo, la participación ciudadana, por medio de la cual desea ampliar y democratizar la toma de decisiones, es solo para la población que asuma sin remedio dicha visión de la sostenibilidad.

El «desarrollo sostenible a la tica» se convirtió así en el sinónimo y monopolio de la protección ambiental en el país, un agente aglutinante de toda su visión desde lo nacional, lo regional y global, pero que oculta bajo una retórica conservacionista intereses económicos (MREC, 1996, pp.64-66). Sobre esto apunta Cuello:

En la formación del pensamiento costarricense sobre la conservación y el desarrollo sostenible, la Estrategia Mundial para la Conservación, de la Unión Internacional para la Conservación de la Naturaleza (UICN) en 1980, y el informe de la Comisión Mundial de Medio Ambiente y Desarrollo, de la ONU en 1987 (Nuestro Futuro Común), han jugado un rol de primerísima importancia (Cuello, 2011, p.188).

Con lo cual se deriva su uso nacional del contexto global, es decir, de lo que el sistema internacional ha definido como desarrollo sostenible y sus instrumentos. Sin embargo, la referencia al escenario mundial no es neutral y su relación es aún más compleja, ya que no es una imposición desde las superpotencias y sus organismos internacionales sobre los países periféricos o así llamados en desarrollo, tal como suelen considerar muchas perspectivas de las Relaciones Internacionales y Geopolítica.

Tomando en cuenta todo lo anterior, se elabora el Mapa Conceptual 1. En el cual se ilustra cómo es el contexto del sistema internacional y su diseño neoliberal, la base de la elaboración del «desarrollo sostenible a la tica», convirtiéndose en una historia local que interpreta y acepta dicho diseño. De ahí, su carácter heterárquico, el cual si bien acepta incuestionablemente el contenido económico-político del desarrollo sostenible, este se hace por medio de la 
particularización nacional y local de sus postulados. Es decir, la creación del «desarrollo sostenible a la tica» es una suerte de "respuesta" (nunca una "pregunta") a nivel nacional para insertarse/funcionar exitosamente en ese diseño global.

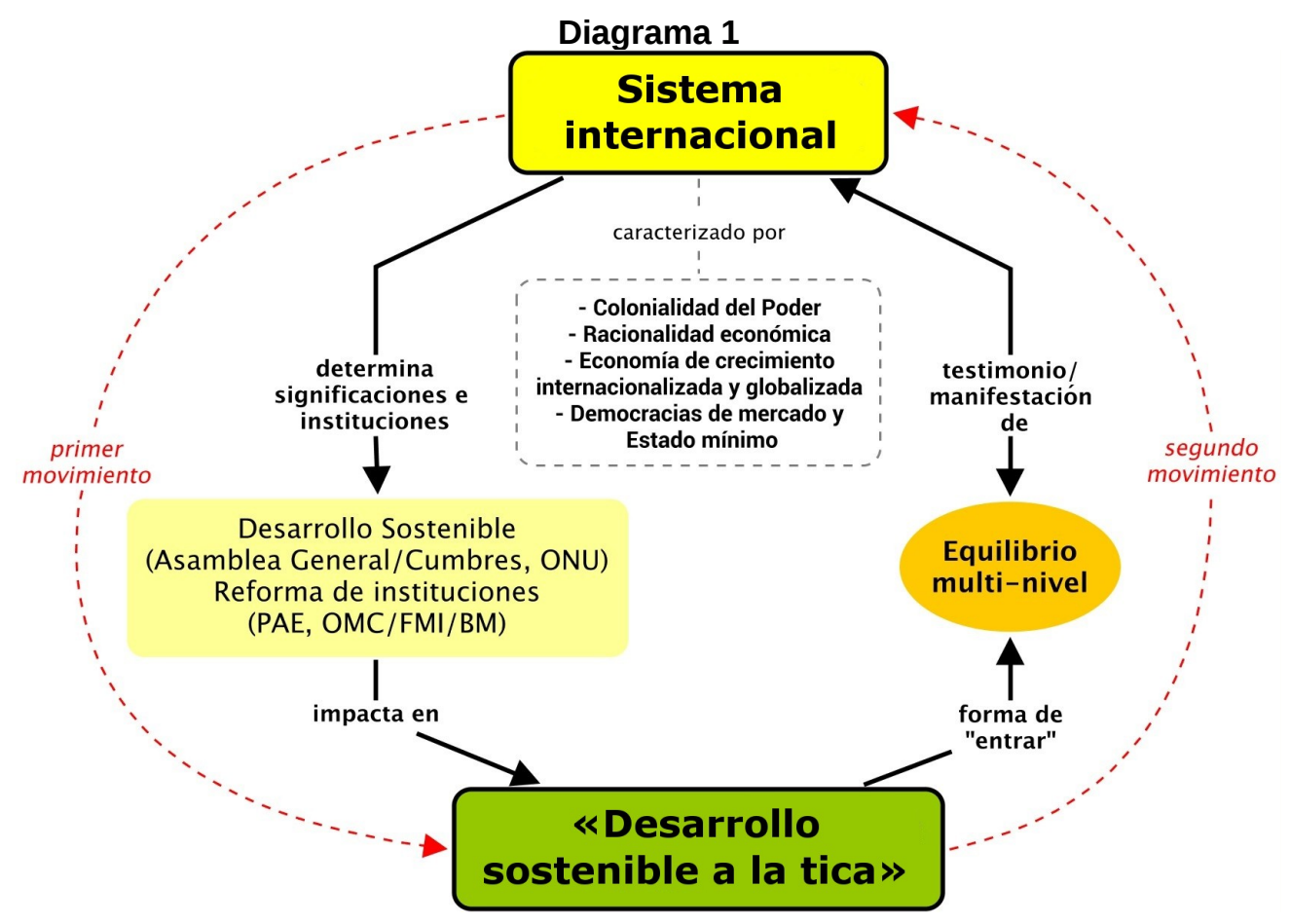

Se destaca en el Mapa Conceptual N1 la existencia de dos movimientos: el primero "externo-interno", el cual viene desde los temas centrales de Occidente y sus potencias, del diseño neoliberal, con la complicidad de los foros económico-políticos alrededor de la ONU, OMC, FMI y BM; el segundo movimiento, "interno-externo", la respuesta al sistema que articula el dicho desarrollo. Su objetivo lo confirma el Canciller Fernando Naranjo, que junto a Luis Guillermo Solís (enviado especial para Centroamérica) fueron las cabezas del equipo internacional del Ministerio de Relaciones Exteriores y Culto (MREC) de la Administración Figueres Olsen, cuando afirma:

El propósito que anima la nueva visión de la política internacional de la Administración Figueres Olsen es un compromiso claro de dotar a Costa Rica de los instrumentos diplomáticos que le permitan en el siglo venidero asumir a plenitud las oportunidades de un mundo cada 
vez más competitivo e integrado, que demanda de nuestro país una

eficiencia sin precedentes en el concierto de las Naciones (MREC, s.f., p.3).

Se puede observar como existe una retórica que vincula el destino nacional al diseño neoliberal. Primero, se anuncia la ruptura con un esquema anterior, al llamar a una "nueva visión", y, luego, se insta a aceptar la integración mundial como inevitable (como si de un parafraseo de Thatcher se tratara), ya que este nos demanda la "inserción" a un "mundo cada vez más competitivo e integrado", por medio de los "instrumentos diplomáticos". Siendo la integración y competitividad referencias a las dinámicas económicas surgidas de la globalización e internacionalización de la economía de crecimiento.

De esta manera, el uso del desarrollo sostenible se presenta como sinónimo de protección ambiental y como agente aglutinante de la Administración. Si bien es una elección de sus personeros, viene dado del juego geopolítico y geoeconómico global, donde fueron otros sujetos y otros Estados los que le dieron la importancia a la degradación de las condiciones ambientales del mundo, son las superpotencias y sus organismos internacionales las que terminan por definir la orientación que este va a tener.

En consecuencia, el desarrollo sostenible no fue finalmente aquella dimensión, que de forma holística e integral abarcara las relaciones humanidad-naturaleza, y que así cambiara los diseños globales de desarrollo. Su principal meta es el crecimiento económico y no el bienestar de la humanidad y su simbiosis con todas las formas de vida del planeta. Por el contrario, se tomó el ambiente como una variable métrica más, en palabras de Figueres Olsen, para seguir las señales del mercado (como si fuera un ente extrasocial) procurando desperdiciar y dañar menos a la naturaleza. Imponiendo entonces una nueva máxima, se protege o se conserva la biodiversidad siempre y cuando esto sea rentable económicamente.

\section{Práctica del «desarrollo sostenible a la tica»}

Las acciones específicas de la Administración Figueres Olsen que buscaron operacionalizar el «desarrollo sostenible a la tica» son divididas por el propio gobierno en: (a) enfrentar sesgos económicos para valorizar los recursos naturales; (b) contener o revertir la degradación ambiental provocada por el mal manejo de desechos y sobreexplotación; (c) impulsar cambios en la administración de las instituciones públicas. Resulta interesante observar cómo en este caso se deja la retórica y se visualiza lo que ya se comentado, es por medio de lo económico que se busca salvaguardar el medio ambiente, visto este último como un recurso o un capital. Lo que convierte al diseño neolibe- 
ral y a la economía de crecimiento en piedras angulares indestructibles e incuestionables a las que solo se hay que ajustar.

El mismo gobierno es quien enumera los siguientes logros que pasamos a comentar (MIDEPLAN, 1998):

- Se crea el código para la ECODES por medio de Ley Orgánica del Ambiente, no obstante concluye Cuello (2011) que esta posee un carácter eminentemente antropocéntrico, en donde el ambiente es 'un patrimonio' de las personas para ser usado en forma sostenible, [sin poseer] ningún principio que reivindique el valor intrínseco de la naturaleza y su derecho a existir al margen de lo humano, [haciendo que] los cambios y evolución de los sistemas de la naturaleza se [midan] sólo en función de los indicadores económicos (Cuello, 2011, pp.247248, énfasis propio).

- Bajo esta visión, se centralizan en el Sistema Nacional de Áreas de Conservación (SINAC) las diferentes instituciones que administraban las áreas de conservación e incorporan los consejos locales y regionales para incluir a las comunidades que asuman el «desarrollo sostenible a la tica».

- Se firma la Alianza para el Desarrollo Sostenible (ALIDES) en el marco del SICA durante la Cumbre Ecológica de Volcán Masaya (Octubre de 1994). No obstante, su principal fruto no fueron políticas ambientales, sino homologar criterios para lograr así firmar la Declaración Conjunta Centroamericana-USA (CONCAUSA), la cual representa un nuevo esquema para las relaciones y cooperación entre Estados Unidos y Centroamérica de forma multilateral, base material para la posterior negociación y firma del Tratado de Libre Comercio (DR-CAFTA).

- De la ALIDES también se desprende el "Convenio para la conservación de la biodiversidad y protección de áreas silvestres", el cual promovió la bioprospección mediante investigación privada y venta de derechos de investigación de la biodiversidad (Decreto Ejecutivo No. 49-95, de Septiembre de 1995, Gaceta No. 198).

- Por ejemplo, de los resultados de esta iniciativa es el acuerdo entre el Instituto Nacional de Biodiversidad (INBio) y la transnacional MERCK, para la recolección de muestras de plantas e insectos para la elaboración de extractos y de muestras ambientales, los cuales son ensayados por la transnacional en sus laboratorios para determinar su uso potencial en la industria farmacéutica y veterinaria.

- En política forestal, se introdujeron los "Certificados para la protección del bosque", que tenían como propósito retribuir al propietario por no 
usar los bosques para explotación de tipo maderera, pero dejaban sin regulación el uso del suelo, de plantas, etc. Al mismo tiempo que fueron usados por la Administración para su venta a países industrializados como Servicios Ambientales de "eliminación del carbono atmosférico", con los cuales estas potencias, contrario a presionar a sus industrias a reducir sus emisiones de dióxido de carbono, solo "compran" (mediante la plantación o la conservación de bosques) a los países periféricos para que reduzcan las cantidades de carbono.

Efectivamente, se observa nuevamente la manera en la cual la naturaleza es utilizada bajo una racionalidad económica y una lógica (bio)colonial, ya que no solo se resalta la afirmación de Cuello (2011), de que la naturaleza es medida bajo los índices económicos, sino que reside en el fondo de estas medidas la racionalidad económica, en la cual la capacidad de lucro impera sobre la conservación y protección ambiental (Leff, 2004).

Por ejemplo, en el último punto sobre "venta" de aire. Resulta evidente que es más barato a los países industrializados "comprar" este aire que modificar sus procesos de fabricación para reducir sus emisiones de carbono; al punto que se logra incorporar dichas compras al "Protocolo de Kioto" como "Certificados transferibles de mitigación". Comercializados internacionalmente por medio del Ministerio de Recursos Naturales, Energía y Minas a cargo de René Castro.

Otras acciones concretas que se elaboraron fueron:

- Importación y consumo de "gasolina súper", un diesel más limpio y el programa ECOMARCHAMO para el control de emisiones, además, la capacitación a los mecánicos por medio de Instituto Nacional de Aprendizaje. Ya que el $70 \%$ de la contaminación es de origen automotriz y se concentra en el Gran Área Metropolitana, sin embargo, se dejó de lado el aumento desproporcionado de vehículos (de primera y segunda mano) en el país, y se enfoca en la reducción (no eliminación) de contaminantes.

- Control de afluentes impulsada por medio del programa "Bandera ecológica" como incentivo a las comunidades por la protección del ambiente, y "Bandera azul" a los hoteleros, cámaras de turismo y comunidades costeras, al satisfacer una calidad del agua apta para consumo humano, limpieza de playas, tratamiento de aguas residuales, etc. Nótese que en el caso de los grandes propietarios de tierras se utiliza el Pago de Servicios Ambientales, mientras que en estos casos se les entrega un reconocimiento simbólico, una bandera.

- Para el manejo de desechos sólidos, se intentó la modernización de la legislación, el desarrollo de proyectos y el fortalecimiento institucio- 
nal, como el fallido cierre de Río Azul y la promoción de la iniciativa privada en la construcción y administración de rellenos sanitarios.

- Se dio importancia a la satisfacción de la demanda energética por medio del fortalecimiento de actividades para la promoción de la eficacia de su uso y el desarrollo de fuentes de energía renovable bajo un esquema mixto de cogeneración de electricidad con iniciativa privada. Como resultado, la generación privada de electricidad se multiplicó por diez entre 1994 y 1997 (MIDEPLAN, 1998).

- Se fomenta el ecoturismo, sin embargo, otros estudios apuntan a que no existe una correlación por parte de la inversión en problemas ambientales y la consolidación del país como destino turismo natural (Avendaño, 2005, p.62).

Los indicadores que buscan medir objetivamente el desarrollo sostenible no aparecieron sino hasta después de la Administración, por ejemplo, el primer informe "GEO Costa Rica: una perspectiva sobre medio ambiente" del Instituto Nacional de Estadística y Censo se publicó en 2002, es decir, 4 años más tarde de la salida de Figueres Olsen en 1998, justo en ese año (1998) el propio Informe de Estado de la Nación decía, al evaluar la calidad de información sobre ambiente, que:

Persisten limitaciones para el acceso a series estadísticas fiables, consistentes y actualizadas para el análisis del desempeño nacional en materia ambiental. Las decisiones en este campo se toman con escasa información... No se cuenta con datos recientes, el acceso a los pocos existentes es difícil, no hay continuidad en las variables que se mide, la información está dispersa, y la que está disponible proviene de fuentes y metodologías diversas, lo que dificulta la realización de comparaciones (Estado de la Nación, 1999, p.167).

Asimismo, muchos indicadores que se han creado en función de este tipo de desarrollo sostenible, mantienen una medición econométrica del ambiente, al definirlo como "recursos naturales" o "capital ambiental", conceptos que distan mucho de la visión holística que debería tener esta nueva relación naturaleza-sociedad, como complementos del caleidoscopio de la vida en el planeta. Claro está, esto sin despreciar mediciones tan importantes como las que nos permiten evaluar la relación medio ambiente y salud humana por medio de la cuantificación de elementos contaminantes en los suelos, aguas y aires, presentes en las tierras cultivables, los alcantarillados y cuencas hi- 
drográficas, así como en las vías de comunicación con alto impacto, especialmente concentrados en los cascos urbanos.

De los pocos datos con los que se pueden contar para el periodo, se encuentra que no hubo cambios (o hubo muy pocos) en: reserva natural estricta, parques nacionales, áreas de manejo de hábitat/especies y áreas protegidas con recursos manejados, entre otros (FLACSO, 2006). Al mismo tiempo, sobre polución, como se muestra en la tabla 1, se registran disminuciones en el periodo, sin embargo, hacia el final del cuatrienio hubo un repunte en emisión de partículas, de $\mathrm{SO}_{2}, \mathrm{NO}_{x}$, Hidrocarburos, $\mathrm{CO}, \mathrm{CO}_{2}, \mathrm{CO}_{2}$ por combustibles fósiles, fabricación de cemento y por procesos industriales, así como por combustibles fósiles líquidos. Finalmente, solo destaca la disminución en emisiones de CFC.

\begin{tabular}{|c|c|c|c|c|c|}
\hline \multicolumn{6}{|c|}{$\begin{array}{c}\text { Tabla } 1 \\
\text { Indicadores Medioambientales (1994-1998) }\end{array}$} \\
\hline Indicador & 1994 & 1995 & 1996 & 1997 & 1998 \\
\hline Emisiones de partículas* & 0,3 & 0,3 & 0,3 & 0,3 & 0,5 \\
\hline $\begin{array}{l}\text { Emisiones de Anhídrico } \\
\text { sulforoso }\left(\mathrm{SO}_{2}\right)^{*}\end{array}$ & 11,8 & 12,3 & 10,8 & 10,6 & 13,1 \\
\hline $\begin{array}{l}\text { Emisiones de Oxido de } \\
\text { nitrógeno }\left(\mathrm{NO}_{x}\right)^{*}\end{array}$ & 36,2 & 37,0 & 36,3 & 36,6 & 42,5 \\
\hline Emisiones de Hidrocarburos* & 5,0 & 6,5 & 7,7 & 8,3 & 8,3 \\
\hline $\begin{array}{l}\text { Emisiones de Monoxido de } \\
\text { Carbono }(\mathrm{CO})^{\star}\end{array}$ & 144,0 & 156,2 & 160,5 & 164,3 & 185,6 \\
\hline $\begin{array}{l}\text { Emisiones de Dióxido de } \\
\text { Carbono }\left(\mathrm{CO}_{2}\right)^{\star a}\end{array}$ & 5,241 & 4,866 & 4,738 & 4,974 & 5,028 \\
\hline $\begin{array}{l}\text { Emisiones de Dióxido de } \\
\text { Carbono }\left(\mathrm{CO}_{2}\right) \text { por procesos } \\
\text { industriales }\end{array}$ & $6.336,3$ & $6.528,9$ & $6.438,0$ & $6.840,5$ & $10.639,8$ \\
\hline $\begin{array}{l}\text { Emisiones de Dióxido de } \\
\text { Carbono }\left(\mathrm{CO}_{2}\right) \text { por } \\
\text { combustibles fósiles y } \\
\text { fabricación de cemento }\end{array}$ & 1.428 & 1.326 & 1.291 & 1.355 & 1.363 \\
\hline $\begin{array}{l}\text { Combustibles fósiles } \\
\text { líquidos*a }\end{array}$ & 1.300 & 1.208 & 1.178 & 1.227 & 1.216 \\
\hline Consumo de CFCs ${ }^{\star c}$ & 184.0 & 158.5 & 497.2 & 94.8 & -204.2 \\
\hline
\end{tabular}


Asimismo, se puede anotar en lo que respecta a los cuatro equilibrios que había planteado la Administración: (a) equilibrio político-institucional, que buscó la amplitud democrática en la toma de decisiones, pero por medio de la aceptación irremediable de esta visión del desarrollo sostenible; (b) equilibrio de la estructura social, que pretende la integración interna, la cual como la mayoría de los discursos políticos de toma de posesión, solo queda como referencia vacía a un apoyo sin cuestionar el fondo del sistema y su élite; (c) equilibrio económico, que se decía era para alcanzar largos periodos de progreso material sostenido, pero en el fondo buscaba la consolidación de las reformas institucionales pactadas en el bipartidismo; y el (d) equilibrio ambiental, según el cual pretendía una armonía entre la base natural y la vida social, siempre filtrada y limitada bajo las lógicas (bio)coloniales y la racionalidad económica mencionadas.

Pese a este panorama, la Administración consideró su gestión ambiental un éxito, según muestra la intervención de Figueres Olsen en la Cumbre Río+5 (1997), al referirse sobre el cumplimiento o no del "Programa 21" destaca que el «desarrollo sostenible a la tica» ha avanzado: (a) en lo económico, mejorando los balances macroeconómicos y propiciando el ahorro y la posibilidad de inversión; (b) en lo social, inversión en bienestar para mejorar cobertura y calidad de servicios; (c) en lo ambiental, creando una alianza con la naturaleza para poder aprovechar tanto hoy como en el futuro los recursos (Figueres Olsen, 1998). Así pues, como se hizo costumbre, el ambiente queda relegado al cumplimiento de factores económicos.

En síntesis, la retórica ambientalista del gobierno del PLN de Figueres Olsen sirvió para promover el «desarrollo sostenible a la tica», ya que, como se ha mencionado, la naturaleza es conservada siempre y cuando exista un lucro por ello; esto es capitalismo ecológico en palabras de Gómez (2012). La principal consecuencia, sin embargo, es que dicho discurso sobre las políticas ambientales se convirtió en política de Estado, lo que significa que se vuelve no solo la versión oficialista de este gobierno, sino que también la versión institucionalizada por sobre la cual los demás gobiernos elaboran su discurso y su práctica sobre la naturaleza.

Esto es particularmente evidente en los gobiernos liberacionistas, como lo han sido las políticas de "Paz con la naturaleza" de la segunda Administración Arias Sánchez (2006-2010), el uso de la imagen internacional del país como destino turístico verde y el discurso de justicia ambiental de la Administración Chinchilla Miranda (2010-2014) en el Rio+20. No sin olvidar las pretensiones políticas del mismo Figueres Olsen con su proyecto país "Vía costarricense" (Rodríguez, 2012). No obstante, como telón de fondo están las disputas socio-ambientales en temas como la minería a cielo abierto, la protección y distribución del agua como dominio público, la generación eléctrica y megarepresas, la cacería y pesca deportiva, los proyectos turísticos dentro y fuera de áreas de protección, la construcción de la Trocha 1856 y la ausen- 
cia de una educación ambiental coherente con la protección del medio ambiente.

Finalmente, se debe apuntar sobre el comportamiento de las élites gubernamentales y las élites empresariales, donde es común la movilidad entre sus funcionarios, por ejemplo, el mismo Figueres Olsen pasó de ser presidente de Costa Rica después de participar de varias fundaciones, empresas y organismos de la ONU, a ser Director Ejecutivo y posteriormente Consejero Delegado (CEO, por sus siglas en inglés) del Foro Económico Mundial, que deja después del escándalo de ALCATEL, y asume, además, un papel dinámico dentro de organizaciones internacionales que promueven el desarrollo sostenible como única salida a la depredación ambiental. Esto evidencia la complicidad para dar contenido al desarrollo sostenible y sus versiones locales, como el «desarrollo sostenible a la tica», entre las élites gubernamentales, los organismos internacionales, empresas y corporaciones transnacionales en un nuevo traslado, privatización o desnacionalización del ámbito público/nacional al privado. Como ya dejan ver las primeras propuestas de la así llamada "economía verde" (Unmüßig, 2012).

\section{Bibliografía}

Alimonda, H. (Coord.) (2011). La naturaleza colonizada: ecología política y minería en América Latina. Buenos Aires: CLACSO \& CICCUS.

Avendaño, I. (2005). La relación ambiente y sociedad en Costa Rica: entre gritos y silencios, entre amores y odios. San José: Editorial UCR.

Cajigas-Rotundo, J.C. (2011). La (Bio)Colonialidad del Poder: cartografías epistémicas en torno a la abundancia y la escasez. Youkali, 11. 59-74.

Castro-Gomez, S. (2007). Michel foucault y la colonialidad del poder. Tabula Rasa, 6, 153-172.

Cuello Nieto, César. (2011) Desarrollo Sostenible y experiencias costarricenses. Heredia: EUNA.

Estado de la Nación (1999). Estado de la Nación en Desarrollo Humano Sostenible: un análisis amplio y objetivo sobre la Costa Rica que tenemos a partir de los indicadores más actuales 1998. San José: Proyecto Estado de la Nación.

Figueres Olsen, J.M. (1994a). El camino de las oportunidades está abierto: discurso del Ingeniero José María Figueres en la Ceremonia de Traspaso del Poder Ejecutivo, en el Estadio Nacional. Domingo 8 de mayo de 1994. San José: Ministerio de Información y Comunicación. 
Figueres Olsen, J.M. (1994b). De la pacificación al Desarrollo Sostenible: Discurso del señor Presidente de Costa Rica, Ingeniero José María Figueres, en la apertura de la XV Reunión de Presidentes Centroamericanos, en Guácimo, Costa Rica, el 18 de agosto de 1994. San José: Ministerio de Información y Comunicación.

Figueres Olsen, J.M. (1998). Construimos el puente hacia el nuevo milenio. San José: Ministerio de Información y Comunicación.

FLACSO (2006). Centroamérica en cifras: 1980-2005. San José: FLACSO \& UCR.

Fotopulos, T. (2002). Hacia una democracia inclusiva: la crisis de la economía de crecimiento y la necesidad de un nuevo proyecto liberador. Montevideo: Nordan-Comunidad.

Gómez , A. (2012). La colonialidad de la naturaleza: reflexiones a partir del caso mexicano. En: Marañón-Pimentel, B. (Coord.) Solidaridad económica y potencialidades de transformación en América Latina: una perspectiva descolonial. Buenos Aires: CLACSO.

Gudynas, E. (2003). Ecología, economía y ética del Desarrollo Sostenible. Bolivia: ICIB \& ANCB.

Hybel, A. y Lynch, R. (1994). Política Exterior de los Estados Unidos hacia América Latina. Polémica: Revista Centroamérica de Ciencias Sociales, 4, 41-53.

Leff, E. (1994). Ecología y capital: racionalidad ambiental, democracia participativa y desarrollo sustentable. México: Siglo XXI Editores.

Leff, E. (2004). Racionalidad ambiental: la apropiación social de la naturaleza. México: Siglo XXI Editores.

MIDEPLAN. (1998). Gobernando en Tiempos de Cambio. San José: MIDEPLAN.

Mignolo, W. (2003). Historias locales/diseños globales: colonialidad, conocimientos subalternos y pensamiento fronterizo. Madrid: Akal Ediciones. Ministerio de Relaciones Exteriores y Culto (1997) Memoria 1996-1997. San José: Ministerio de Relaciones Exteriores y Culto.

Ministerio de Relaciones Exteriores y Culto (s.f.). Centro América: hacia una política exterior común. San José: Ministerio de Relaciones Exteriores y Culto. 
Ministerio de Relaciones Exteriores y Culto. (1996). Memoria 1995-1996. San José: Ministerio de Relaciones Exteriores y Culto.

Molinan, I. y Palmer, S. (1997). Historia de Costa Rica. San José: Editorial UCR.

Monge, G. (Edit.) (1995). Por los Caminos del Desarrollo Sostenible: discursos del señor presidente de la República José María Figueres Olsen. San José: Ministerio de Información y Comunicación.

Monge, C.A. (2013) Instituciones y significantes en las políticas exteriores de Costa Rica con la integración centroamericana: nacionalismo en las administraciones Figueres Olsen y Rodríguez Echeverría dentro del Sistema de Integración Centroamericana (1994 - 2002). Tesis de Licenciatura no publicada, Universidad de Costa Rica, San José, Costa Rica,

ONU. (1987). Nuestro Futuro Común. Mimeo.

ONU. (1989). Resolución 44/228 de la Asamblea General. Mimeo.

ONU. (s.f.). Documentación sobre medio ambiente. Recuperado de http://www.un.org/depts/dhl/spanish/resguids/specenvsp.htm

Quesada M. (1998). Estrategia Nacional de Conservación para el Desarrollo Sostenido (ECODES). San José: Ministerio de Recursos Naturales, Energía y Minas.

Quijano, A. (2000) Colonialidad del poder, globalización y democracia. Mimeo.

Rodríguez, I. (09 de mayo de 2012) Figueres: 'El planeta ya no puede sostenerse como lo hacía antes'. La Nación, p. 21.

Segura, O. (Comp.) (1992). Desarrollo sostenible y políticas económicas en América Latina. San José: DEI.

Stokke, O. y Mármola, L. (1993). Los dilemas del desarrollo sostenible. San José: FLACSO.

Unmüßig, B. (2012) Economía verde: ¿La nueva fórmula mágica? expectativas acerca de la Conferencia Río+20. Zeitschrift Vereinte Nationen, 60(1), 3-9. 